\title{
Evidence from Impedance Spectroscopy that Elevated Dopamine Reduces Mitochondrial Membrane Potential
}

\author{
Mitra RC ${ }^{1,2}$, Villagrán MYS ${ }^{1,2}$, Maric $\mathbf{S}^{1,2}$, Wosik $\mathbf{J}^{2,3}$, Zagozdzon-Wosik $\mathbf{W}^{3}$ and Miller $\mathrm{JH}^{\mathrm{J}} \mathbf{r}^{1,2 *}$ \\ ${ }^{1}$ Department of Physics, University of Houston, Houston, Texas, USA \\ ${ }^{2}$ Texas Center for Superconductivity, University of Houston, Houston, Texas, USA \\ ${ }^{3}$ Department of Electrical and Computer Engineering, University of Houston, Houston, Texas, USA
}

\begin{abstract}
Background: Recent studies suggest that elevated dopamine concentrations reduce mitochondrial membrane potential, potentially contributing to neurological or other disorders. Changes in average membrane potential of suspended cells or organelles can be monitored in real time by measuring impedance of the suspension as a labelfree assay.

Methods/Results: Here we report on measurements of frequency- and time-dependent electrical impedance of mitochondrial suspensions in the presence of various substrates, including dopamine. We find that, during succinateinduced state-2 respiration, addition of either trifluoromethoxy carbonylcyanide phenylhydrazone (FCCP), a known mitochondrial uncoupler (depolarizer), or dopamine at high concentrations cause similar increases in impedance, consistent with a drop in mitochondrial membrane potential.

Conclusion: Our results provide further evidence that dopamine reduces mitochondrial membrane potential, albeit less severely than FCCP, possibly by generating free radicals that partially perforate the mitochondrial inner membrane and/or reduce activity of respiratory complexes. These results suggest that prolonged excess dopamine can impair ATP production, with possible implications in some neurological disorders and suggesting the need for care in the use of dopamine to treat Parkinson's disease or heart failure.
\end{abstract}

Keywords: Mitochondria; Electrical impedance spectroscopy; Dopamine; Mitochondrial dysfunction; Membrane potential; Mitochondrial uncoupler

\section{Introduction}

Mitochondria, often called cellular "powerhouses," perform critical functions that include ATP production, neurotransmitter synthesis and degradation, and apoptosis [1]. Impaired ATP production due to mitochondrial dysfunction [2-4] and dysregulation of signaling molecules [2,4-6] play important roles in neurological and cardiovascular diseases [7]. Dopamine is an organic chemical that functions as a neurotransmitter in the brain and as a chemical messenger (hormone, vasopressor, etc.) in the digestive system, kidneys, and cardiovascular system. The brain contains dopaminergic pathways associated with reward-related cognition and learning, while cardiovascular dopaminergic receptors affect functions such as cardiac contractility and vasodilation. Dopamine dysregulation is implicated in disorders that include Parkinson's disease, schizophrenia, and drug addiction [2,4-6], the latter two of which involve elevated dopamine levels in key brain regions. Dopamine and its analogs have been used to treat neurological disorders such as Parkinson's disease [8,9], and to improve cardiac function in patients with heart failure $[10,11]$. Dopamine metabolism, however, can generate reactive oxygen species that cause oxidative stress [12], and its prolonged use can cause unwanted cardiovascular side effects [9].

Excessive dopamine concentrations may exacerbate mitochondrial dysfunction, which contributes to cardiovascular, neurological, and other illnesses [2,7]. Recent studies suggest that elevated dopamine concentrations reduce mitochondrial membrane potential $[4,13]$. This may be due to damage caused by dopamine-induced oxidative stress [12], such as impaired function of respiratory complexes (complexes I-IV) and/or partial perforation (uncoupling) of the mitochondrial inner membrane. Neuronal disorders, including schizophrenia, Parkinson's disease, and Huntington's disease, often involve mitochondrial defects within the respiratory chain [2]. Impaired ATP production, due to mitochondrial dysfunction, also plays a key role in the energetics of heart failure $[14,15]$.

The mitochondrial membrane potential $\left(\Delta \Psi_{\mathrm{m}}\right)$ drives ATP production, and thus affects the state of activity, health, and/or dysfunction of mitochondrial metabolism [16,17]. In our recent studies, impedance spectroscopy has been shown to be a label-free method of probing temporal changes in $\Delta \Psi_{\mathrm{m}}$, as a tool to help monitor mitochondrial function [18]. Through previous studies by Prodan et al. [19-21], changes in membrane potential were shown, both theoretically and experimentally, to correlate with changes in impedance and dielectric response of suspended cells or organelles. Dielectric or impedance response can thus reveal information about biological function, including changes in mitochondrial membrane potential [18]. Potential applications include probing cellular function in vitro $[22,23]$ or even monitoring functional activity in live tissue. Certain frequency ranges $\left(\sim 10^{6} \mathrm{~Hz}\right)$ may allow penetration of the cellular plasma membrane to monitor bioenergetic activity of mitochondria within whole cells or live tissue [24].

A key hypothesis here is that chemicals, or substrates, which cause changes in mitochondrial membrane potential, will correspondingly

*Corresponding author: John $\mathrm{H}$ Miller, Department of Physics, University of Houston, Houston, Texas, USA Tel: +1-713-743-8257; E-mail: jhmiller@uh.edu

Received April 06, 2017; Accepted April 12, 2017; Published April 22, 2017

Citation: Mitra RC, Villagrán MYS, Maric S, Wosik J, Zagozdzon-Wosik W, et al. (2017) Evidence from Impedance Spectroscopy that Elevated Dopamine Reduces Mitochondrial Membrane Potential. J Biosens Bioelectron 8: 242. doi: 10.4172/2155-6210.1000242

Copyright: @ 2017 Mitra RC, et al. This is an open-access article distributed under the terms of the Creative Commons Attribution License, which permits unrestricted use, distribution, and reproduction in any medium, provided the original author and source are credited. 
Citation: Mitra RC, Villagrán MYS, Maric S, Wosik J, Zagozdzon-Wosik W, et al. (2017) Evidence from Impedance Spectroscopy that Elevated Dopamine Reduces Mitochondrial Membrane Potential. J Biosens Bioelectron 8: 242. doi: 10.4172/2155-6210.1000242

affect the impedance of a mitochondrial suspension over certain frequency ranges. This hypothesis is motivated by predictions [21] correlating membrane potential, amount of internal negative charge, polarizability of each suspended cell or organelle, and dielectric response. As the membrane potential and internal charge magnitude increase, the low- to mid- frequency dielectric response of the suspension also increases, while the impedance decreases. The current work: a) tests whether impedance changes correlate with expected changes in mitochondrial membrane potential induced by substrates whose effects are known (succinate, FCCP, etc.), and b) then uses impedance response as a tool to explore whether excess concentrations of a novel substrate, in particular the neurotransmitter dopamine, can adversely affect mitochondrial membrane potential in a fashion similar to that of the known uncoupler FCCP.

\section{Materials and Methods}

\section{Circuit model of mitochondrial suspension}

Impedance measurements were performed on mitochondria suspended in buffer solution, detailed in the next section, sandwiched between two electrodes in a parallel plate capacitor configuration. The suspension can thus be modeled as a leaky capacitor, consisting of a capacitance $C$ in parallel with a conductance $G$, as shown in Figure 1. Temporal changes in total impedance largely reflect properties of the suspension itself, e.g., due to changes in mitochondrial membrane potential, since any cable impedance remains constant, while electrode effects are minimized at frequencies of $\sim 100 \mathrm{kHz}$ or higher [20].

The conductance, resistance, and capacitance of the mitochondrial suspension (or test medium) are thus given by:

$$
G=\frac{\sigma A}{d}, R=\frac{1}{G}, C=\frac{\epsilon_{r} \epsilon_{0} A}{d}
$$

where $\sigma$ is the electrical conductivity, $A$ represents the area of the electrodes, $R$ is the resistance, $\epsilon_{0}$ is the permittivity of free space, $\epsilon_{r}$ is the (extremely large [21]) relative dielectric response of the suspension or medium, and $d$ is the spacing between electrodes. The complex impedance $Z$ of the circuit is then given by:

$$
Z=\frac{1}{G+i \omega C}=\frac{R}{1+i \omega R C}=\frac{R[1-i \omega R C]}{1+\omega^{2} R^{2} C^{2}}
$$

while its magnitude is given by:

$$
|Z|=\frac{R}{\sqrt{1+\omega^{2} R^{2} C^{2}}}
$$

If $\omega^{2} R^{2} C^{2} » 1$, then $|\mathrm{Z}| \approx 1 / \omega C$. Thus, in this limit, if $C$ decreases (e.g., due to decreasing membrane potential of the mitochondria [21]) then $|Z|$ increases. This will also be true in general if $R$ remains constant. Moreover, the conductivity $\sigma$ can increase with membrane potential [19], also causing $Z$ to decrease since $R=d / \sigma A$ decreases. In summary

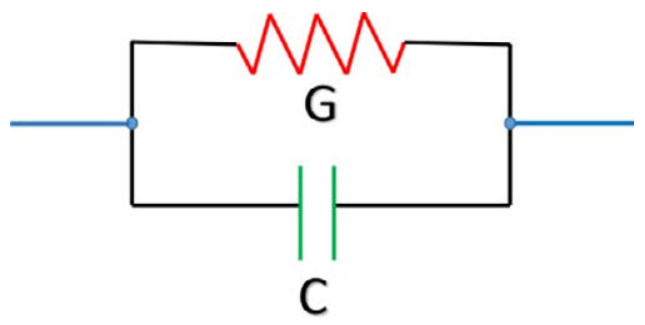

Figure 1: Circuit model of mitochondrial suspension. a sudden increase in mitochondrial membrane potential will tend to cause the measured impedance to decrease, and vice versa.

\section{Chemical substrates}

There are two key chemicals used as substrates in this work: first, trifluorocarbonylcyanide phenylhydrazone (FCCP), molecular formula $\mathrm{C}_{10} \mathrm{H}_{5} \mathrm{~F}_{3} \mathrm{~N}_{4} \mathrm{O}$ with a molecular weight of $254.17 \mathrm{~g} / \mathrm{mol}$; and, second, dihydroxyphenylethylamine (dopamine), with the molecular formula (HO) ${ }_{2} \mathrm{C}_{6} \mathrm{H}_{3} \mathrm{CH}_{2} \mathrm{CH}_{2} \mathrm{NH}_{2}$ and a molecular weight of $153.18 \mathrm{~g} / \mathrm{mol}$. FCCP is $95 \%$ soluble in ethanol and dopamine is water-soluble. For lab uses, dopamine is used in solvent form as dopamine hydrocloride $(\mathrm{HO})_{2} \mathrm{C}_{6} \mathrm{H}_{3} \mathrm{CH}_{2} \mathrm{CH}_{2} \mathrm{NH}_{2} \mathrm{HCl}$. FCCP is a protonophore $\left(\mathrm{H}^{+}\right.$ionophore $)$ and uncoupler of oxidative phosphorylation in mitochondria, which abolishes the linkage between respiratory chain activity and ATP production. In the presence of an electrochemical proton gradient across the membrane, FCCP will become protonated, and thus, pick up a proton on the positive side of the membrane, move across the membrane in the neutral form, and release the proton on the negative side. This FCCP-induced leakage of protons across the inner membrane is well known to reduce mitochondrial membrane potential.

Solutions of dopamine in distilled water were made with various concentrations, since the redox state of dopamine is important. The electrochemical behavior of dopamine strongly depends on the $\mathrm{pH}$ values of the solution. This is determined by measuring the $\mathrm{pH}$ of dopamine for each concentration $[25,26]$. As shown in Table 1 , the $\mathrm{pH}$ range indicates that dopamine coexists in all three forms: cationic, anionic, and zwitterionic. All chemicals used in this study were purchased from Sigma Chemical Co. (St. Louis, MO, USA). Our measurements were affected by the fact that, when dopamine is oxidized to dopamine-o-quinone, two electrons from dopamine are transferred to the electrode and two $\mathrm{H}^{+}$ions stay in solution, which actually contributes to the conductivity variation.

As depicted in Figure 2, both the capacitance and conductivity of dopamine are proportional to concentration. Hence the impedance decreases with concentration, as shown in Figure 3. As will be shown in subsequent sections, however, the observed impedance changes

\begin{tabular}{|c|c|c|}
\hline Temperature $\left({ }^{\circ} \mathbf{C}\right)$ & Concentration & $\mathbf{p H}$ \\
\hline 23.5 & $500 \mathrm{nM}$ & 4.93 \\
\hline 23.0 & $1 \mu \mathrm{M}$ & 5.09 \\
\hline 23.1 & $0.5 \mathrm{mM}$ & 4.13 \\
\hline 23.3 & $1 \mathrm{M}$ & 4.91 \\
\hline
\end{tabular}

Table 1: Temperature and $\mathrm{pH}$ of different concentration of dopamine.

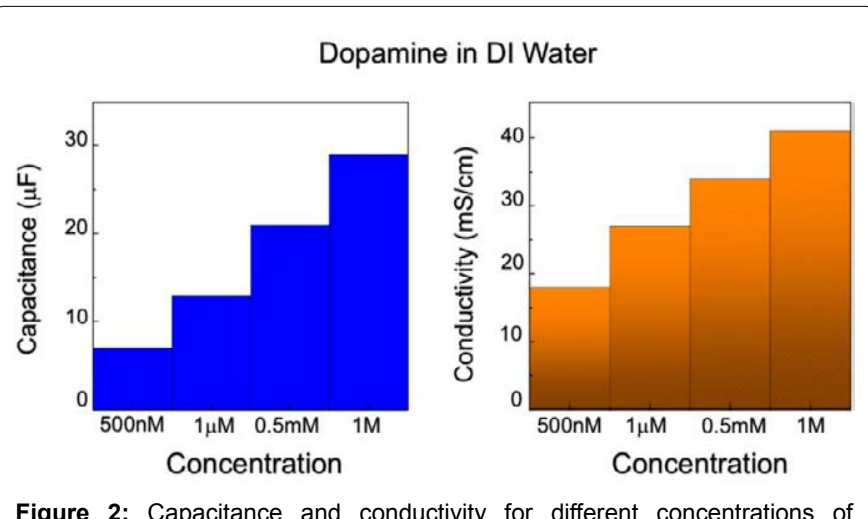

Figure 2. Capacitance and conductivity for different concentrations of dopamine in deionized (DI) water at operating voltage amplitude $10 \mathrm{mV}$ and frequency $2 \mathrm{MHz}$ [26] 


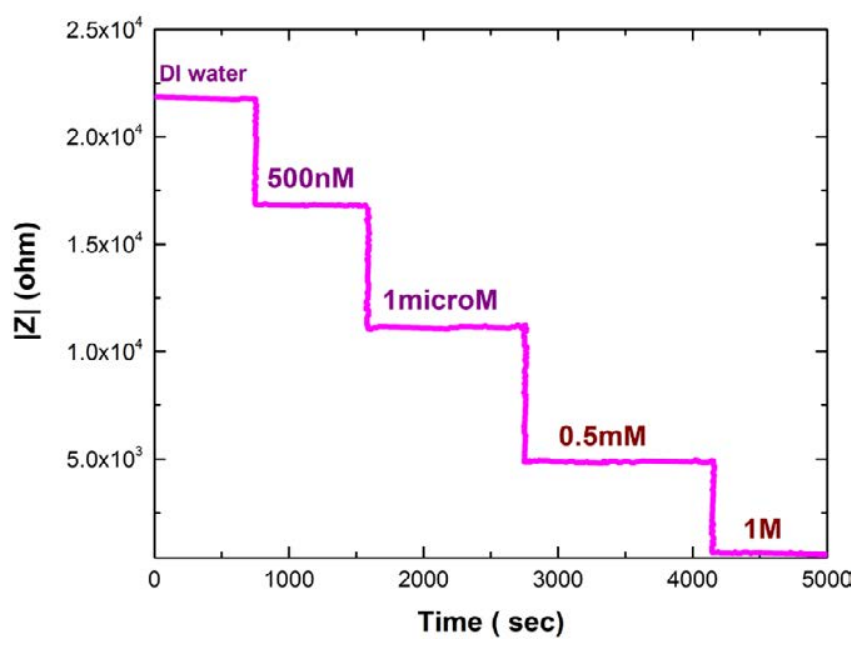

Figure 3: Variation of $|Z|$ for different concentrations of dopamine in deionized (DI) water using a voltage amplitude $10 \mathrm{mV}$ and frequency $2 \mathrm{MHz}$.

of mitochondrial suspensions when adding dopamine are directly opposite to those inferred by dopamine in water alone, supporting the idea that our studies reveal intrinsic changes in mitochondrial properties.

\section{Mitochondrial suspension measurements}

The experimental and animal care protocol was reviewed and approved by the University of Houston Institutional Animal Care and Use Committee (IACUC protocol number 13-016). Each animal employed for tissue harvest of mammalian mitochondria was subjected to high standards of care in accordance with the US Department of Health and Human Services/NIH Public Health Service Policy on the Humane Care and Use of Laboratory Animals. The mice were obtained from Charles River Laboratory and Jackson Laboratory and housed by the University of Houston Animal Care Operations. Each experiment used a minimum of 5 mice hearts. Mitochondria were isolated, using the well-established protocol described in Palmer et al. [27] with minor modifications. The measurements were done on freshly pelleted cardiac mitochondria. Each mouse was isofluoraned for 15 seconds; the heart was extracted, weighed and placed in a glass test tube on ice; the heart was washed four times with Buffer A and then minced on ice. The minced heart was put in $6 \mathrm{ml}$ of Buffer B and then into polytron treatment for 60 seconds. The minced treated heart was then centrifuged at $600 \mathrm{~g}$ for $10 \mathrm{~min}$ at $4^{\circ} \mathrm{C}$, and then the supernatant was saved. The pellet was resuspended in $4 \mathrm{ml}$ of Buffer $\mathrm{A}$ and then centrifuged $600 \mathrm{~g}$ for $10 \mathrm{~min}$ at $4^{\circ} \mathrm{C}$. The supernatant was combined and then centrifuged again at $3000 \mathrm{~g}$ for 15 minutes at $4^{\circ} \mathrm{C}$. Finally, the pellet was resuspended in Buffer C. The buffers used were: Buffer A: 40.08 g $220 \mathrm{mM}$ mannitol (MW=182.17)+23.96 g $70 \mathrm{mM}$ sucrose $(\mathrm{MW}=342.3)+1.05$ g $5 \mathrm{mM}$ Mops $(\mathrm{MW}=209.3)$ in 1 liter of water. Buffer B: $0.076 \mathrm{~g} 2 \mathrm{mM}$ EGTA $(\mathrm{MW}=380.4)+4 \mathrm{~mL}$ of $5 \%$ FAF BSA for $0.2 \%$ BSA in $100 \mathrm{~mL}$ of Buffer A. Buffer C: $0.019 \mathrm{~g}$ $0.5 \mathrm{mM}$ EGTA in $100 \mathrm{~mL}$ Buffer A. Mice cardiac mitochondria were obtained, and the concentration determined typically $\sim 4 \mathrm{mg} / \mathrm{mL}$ using a spectrophotometer, based on the Biuret Method [28,29].

Mitochondrial oxygen consumption was measured with a Clarktype electrode in a Mitocell micro-respirometer (MT200) (Strathkelvin Instruments Limited, Scotland) system. It consists of a 782 oxygen meter, 1302 oxygen electrode, MT200 respirometer, and respirometry software. The MT 200 respirometer consists of a $100 \mu \mathrm{L}$ glass chamber, a transparent polycarbonate plunger, and a magnetic stirrer. The substrates, inhibitors, and uncouplers were injected into the chamber with Hamilton syringes. Respiratory rates of the mitochondrial suspension were measured using the oxygen probe at $25^{\circ} \mathrm{C}$, with continuous stirring. The substrates included $5 \mathrm{mM}$ succinate, which donates electrons to complex II of the electron transport chain. Oxygen consumption rates were expressed in nmoles $\mathrm{O}_{2} / \mathrm{min} / \mathrm{mg}$ dry weight. In Figure 4, we show representative respiration measurements use to compute the respiratory control ratio (RCR) of mitochondria from a mouses hearts. This ratio was used as a general index of the viability of the mitochondrial preparation. Low RCR's ratios raise the possibility of mitochondrial damage. Typical RCR values for well-coupled mitochondria ranged from 3-10.

Impedance spectroscopy was performed on fresh mouse cardiac mitochondria suspended in a respiration buffer solution. The impedance measurements were carried out in an electroporation cuvette with a 4-mm gap- width. Impedance spectra were recorded by the impedance gain/phase analyzer SI-1260 (Solartron Instruments Farnborough, UK). A sinusoidal AC voltage of $10 \mathrm{mV}$ amplitude (peakto-peak) was applied, and ten data points were recorded per frequency decade, chosen to be equidistant on a logarithmic scale up to $10 \mathrm{MHz}$. The current was in the microampere range, preventing mitochondrial stress and death. Temporal effects of added substrates were studied by recording impedance $v s$. time at fixed frequencies.

The data were recorded in real-time, as impedance modulus and phase, from which real and imaginary parts can be inferred. Based on the Biuret method, the mitochondrial sample concentration was typically $\sim 4 \mathrm{mg} / \mathrm{mL}$. Much of the goal of in vitro studies is to replicate, as well as possible, the environment of mitochondria in vivo $[30,31]$. Initially, $4 \mathrm{~mL}$ of respiration buffer was added to the cuvette and the impedance-phase angle values were measured. The respiration buffer was composed of $225 \mathrm{mM}$ mannitol, $75 \mathrm{mM}$ sucrose, $10 \mathrm{mM} \mathrm{KCl}, 10$ $\mathrm{mM}$ Tris- $\mathrm{HCl}$, and $5 \mathrm{mM} \mathrm{KH}_{2} \mathrm{PO}_{4}$, with a $\mathrm{pH}$ 7.2. The buffer served as a control and a baseline for impedance measurements.

\section{Results and Discussion}

Several key findings are summarized here: 1) substrates, known to either increase or decrease mitochondrial membrane potential, cause significant changes in mitochondrial suspension impedance over certain frequency ranges; 2) the impedance changes are consistent with those expected from theoretical predictions $[20,21,32]$; 3 ) in particular,

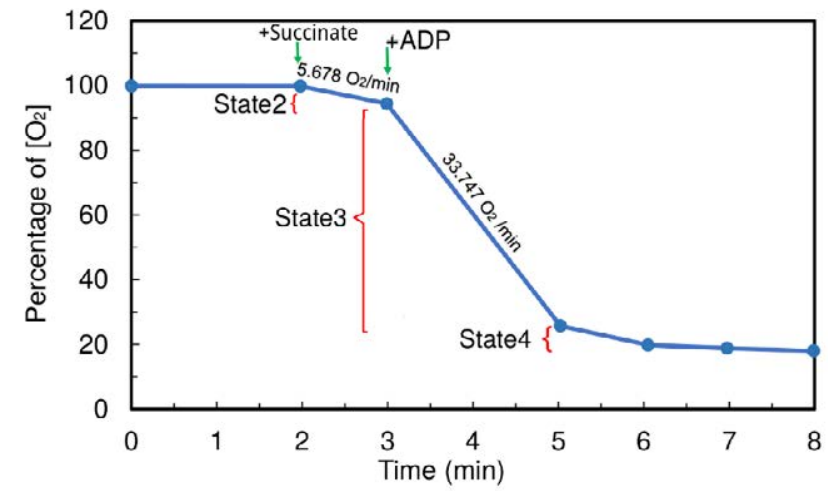

Figure 4: States 1-4 respiration measurements used to compute the $\mathrm{RCR}$ of a suspension of mouse heart mitochondria. Here RCR=State3/ State $4=33.747 / 5.678=5.94$ 
the mitochondrial uncoupler FCCP, known to create a proton leak that greatly reduces membrane potential at high concentrations, causes an increase in impedance at certain frequencies, consistent with predicted drops in overall capacitance and conductance [21]; and 4) the neurotransmitter dopamine, in sufficiently high concentrations, induces changes in impedance similar to those caused by FCCP. This last observation strongly suggests that excess dopamine also reduces mitochondrial membrane potential, either by impairing function of respiratory chain complexes or by creating a proton leak. This may have important implications in the extremes of behavior seen in bipolar disorder or catatonic schizophrenia, where the depressed phase of the former or the catatonic stupor of the latter may result from insufficient ATP production triggered by excess dopamine.

\section{Impedance of mitochondrial suspensions}

The electrical double-layer that forms at the electrode-electrolyte interface creates a spurious low-frequency impedance response [33], which makes data analysis and interpretation difficult at very low frequencies of less than a few $\mathrm{kHz}$. We find however, consistent with our previous measurements [18], that frequencies in the range of 100 $\mathrm{kHz}$ or higher appear to show significant impedance changes that correlate with effects of substrates that greatly affect mitochondrial membrane potential. A Bode plot (Figure 5) shows that the impedance (magnitude) of a respiration buffer decreases with frequency. Figure 5 also shows the effect of adding mitochondria of concentration $3.6 \mathrm{mg} /$ $\mathrm{ml}$ to the respiration buffer. As the buffer with suspended mitochondria becomes more conductive, the impedance decreases. The motion and polarization of charges associated with membrane surfaces and proteins are expected to cause dispersions, with beta dispersions being observed around $1 \mathrm{MHz}$. Addition of succinate at $350 \mathrm{mM}$ further reduces impedance in a manner consistent with an increase in overall dielectric response due to an increase in mitochondrial membrane potential [21].

The presence of plateaus in Figure 5, only with mitochondria present, indicate that these features in dispersion can be attributed mainly to the mitochondrial suspension rather than the buffer. The measurements thus appear to reflect the response from the mitochondrial membranes, charges, and polarizabilities [21,34]. Enclosed charge and polarizability both increase with increasing membrane potential. As polarizability increases, this increases overall dielectric response and bulk capacitance, thereby reducing impedance

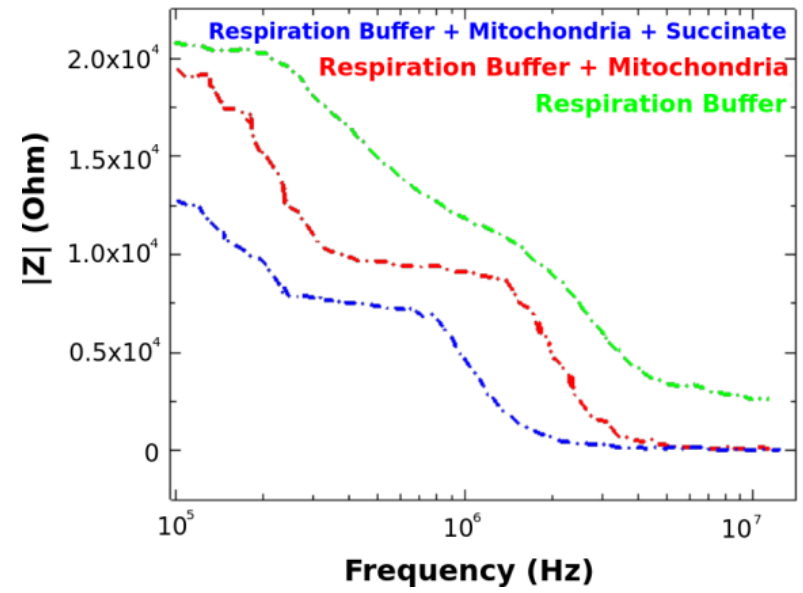

Figure 5: Bode diagram for $|Z|$ vs. frequency for respiration buffer media for different conditions at operating voltage $10 \mathrm{mV}$.
[21]. The small sizes of mitochondria, orders of magnitude smaller than whole cells previously studied [20], shift the impedance changes to higher frequencies. This is due to the more rapid response, to an oscillatory electric field, of charges within these small organelles. The observed decrease in impedance, upon addition of succinate (blue curve in Figure 5), is consistent with an increase in bulk capacitance. This probes the overall dielectric response, which measures the average polarizability per mitochondrion and, thus, is consistent with the expected increase in mitochondrial membrane potential [21] as succinate donates electrons state 2 respiration activates the respiratory chain.

The phase angle (Figure 6) decreases at low frequencies for both the respiration buffer media, with and without mitochondria, and for those activated by succinate. At very low frequencies, the phase angle approaches $-90^{\circ}$ indicating a predominantly capacitive response in Eq. (2). Changes in membrane potential and internal charge affect both alpha and beta dispersion [21], the latter typically in the range $10^{4} \mathrm{~Hz}$ - $10^{7} \mathrm{~Hz}[35,36]$. The increasing charge concentration also leads to a higher conductivity in certain frequency ranges [21] due to motion of charges.

Additional insight is gained by examining the data in terms of capacitance and conductivity, as shown in Figures 7 and 8. The capacitance and dielectric response of the mitochondrial suspension are, in our large dielectric limit, proportional to the average induced electric dipole moment per unit volume Thus, larger dielectric response (i.e., larger bulk capacitance) reflects a larger average membrane potential per mitochondrion. Since the capacitive component of impedance goes as $1 / \omega C$, as $C$ (bulk) increases this contribution to $|Z|$ decreases [21]. The conductivity also increases with frequency (Figure $8)$, consistent with theory [19].

The present study also examined mitochondrial function over a range of succinate concentrations that activate mitochondrial electron transport. Addition of succinate donates electrons to complex II (succinate dehydrogenase) of the respiratory chain. The energy extracted from these electrons is used by complexes III and IV to pump protons across the membrane and build up a mitochondrial membrane potential. As membrane potential increases, the added internal charges augment the mitochondrial polarizability, thereby increasing bulk dielectric response of the suspension and reducing overall impedance.

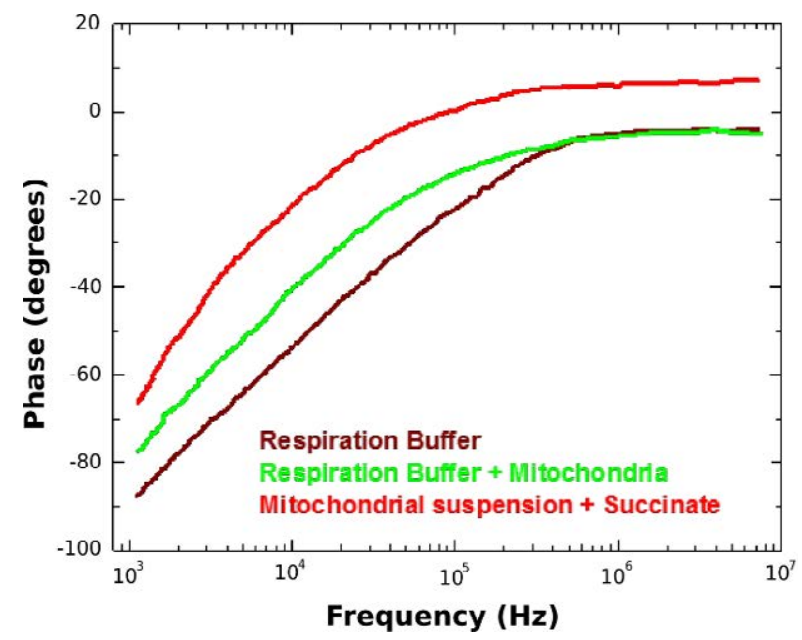

Figure 6: Bode diagram for phase vs. frequency of respiration buffer media for different conditions at operating voltage $10 \mathrm{mV}$. 
Citation: Mitra RC, Villagrán MYS, Maric S, Wosik J, Zagozdzon-Wosik W, et al. (2017) Evidence from Impedance Spectroscopy that Elevated Dopamine Reduces Mitochondrial Membrane Potential. J Biosens Bioelectron 8: 242. doi: 10.4172/2155-6210.1000242

Page 5 of 8

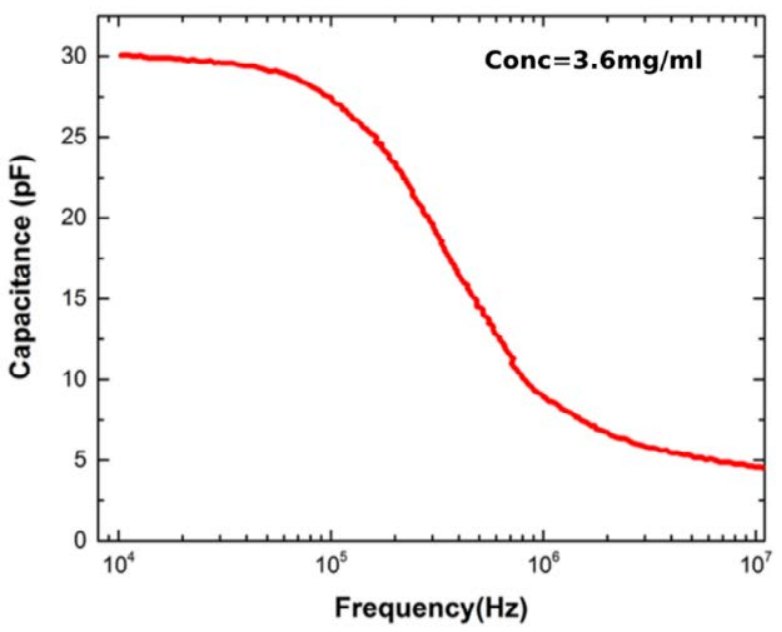

Figure 7: Capacitance vs. frequency for a mitochondrial suspension of concentration $3.6 \mathrm{mg} / \mathrm{mL}$

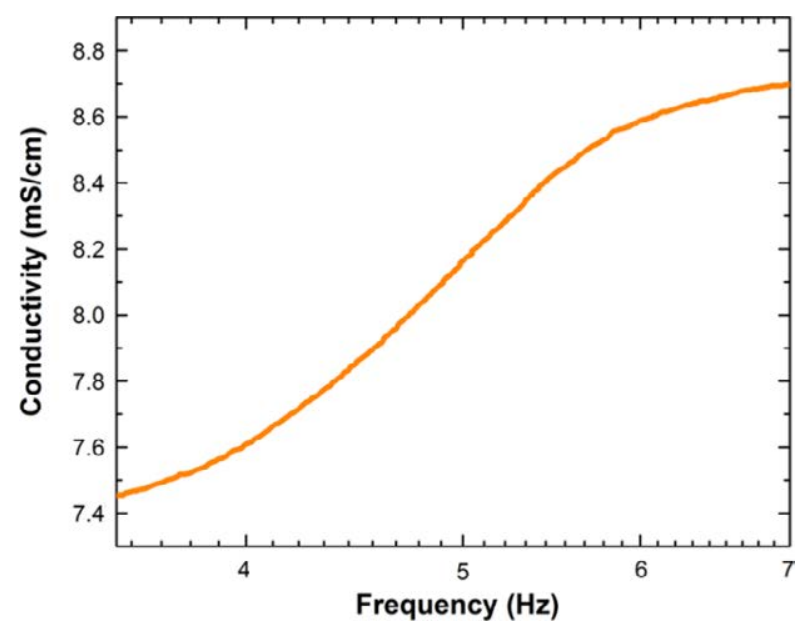

Figure 8: Conductivity vs. frequency for a mitochondrial suspension of concentration $3.6 \mathrm{mg} / \mathrm{mL}$

Further increases in succinate concentration, however, cause only small decreases in impedance, as shown in Figure 9. In this case, the membrane potential attains its maximum value [37], so the decrease in impedance reaches the point of saturation. The results shown in Figure 9 are thus consistent with the expected, initially large and subsequently smaller, changes in membrane potential triggered by added succinate

\section{Effects of FCCP on mitochondrial impedance}

It well established that oxygen consumption rates increase upon addition of the protono-phore FCCP, which causes a collapse of the mitochondrial internal membrane potential at sufficiently high concentrations [38-40]. We find that the impedance of the suspension increases when FCCP is added, as shown in Figure 10. This impedance increase is due to the combined effects of decreasing dielectric response (thus increasing the bulk capacitive reactance of the suspension) and decreasing conductivity (thus increasing its overall resistance). The observed increase in impedance is thus consistent with a reduction of internal mitochondrial charge and with the expected loss of mitochondrial membrane potential. Mitochondrial dysfunction, due to somatic mutations, also reduces our mitochondrial potential as we age
$[3,41,42]$, suggesting possible future applications of impedance-based diagnostic probes.

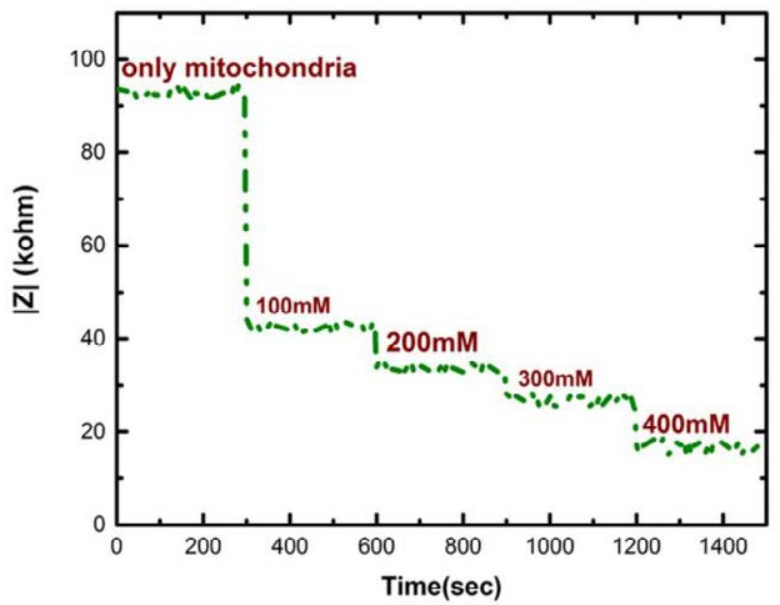

Figure 9: $|Z|$ variation for mitochondria suspension, upon addition of succinate of increasing concentrations, at operating voltage $10 \mathrm{mV}$ and frequency $2 \mathrm{MHz}$.

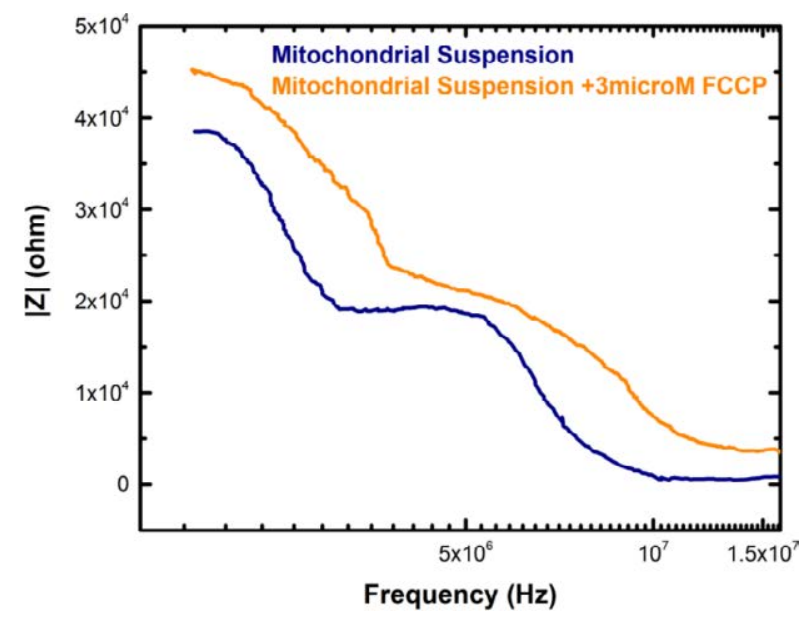

Figure 10: Impedance variation with frequency for FCCP addition to mitochondrial suspension.

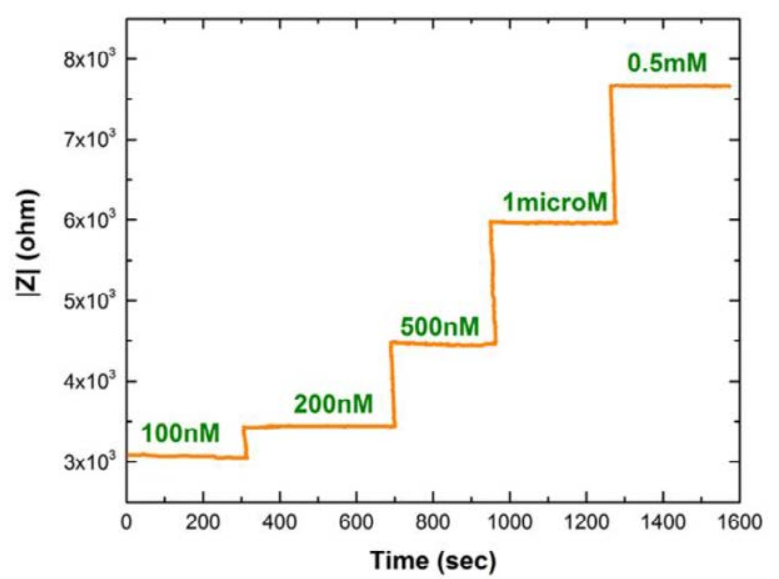

Figure 11: Impedance (at $100 \mathrm{kHz}$ ) vs. time for different concentrations of FCCP added to the mitochondrial suspension. 
Citation: Mitra RC, Villagrán MYS, Maric S, Wosik J, Zagozdzon-Wosik W, et al. (2017) Evidence from Impedance Spectroscopy that Elevated Dopamine Reduces Mitochondrial Membrane Potential. J Biosens Bioelectron 8: 242. doi: 10.4172/2155-6210.1000242

Page 6 of 8

Figure 11 shows temporal impedance changes, at a fixed frequency of $100 \mathrm{kHz}$, upon addition of increasing FCCP concentrations. Previous experiments, adding FCCP to mitochondria and measuring impedance at $100 \mathrm{kHz}, 1 \mathrm{MHz}$, and $10 \mathrm{MHz}$, showed the largest increase in impedance at $100 \mathrm{kHz}$, indicating some increase at $1 \mathrm{MHz}$, and little or no change at $10 \mathrm{MHz}$ [18]. $100 \mathrm{kHz}$ to a few $\mathrm{MHz}$ may thus be the optimum frequency range using impedance to probe temporal changes in membrane potential for mitochondrial suspensions [18]. Electrodeelectrolyte interface effects become problematic if the frequency is too low [20], and require microfabricated designs for reduction of electrode polarization [43], while the frequency falls outside the optimum dispersion region if too high [21].

Higher FCCP concentrations dissipate mitochondrial membrane potential and exacerbate permeation, indicating significant dosedependent uncoupling of mitochondrial respiration [40]. This property is consistent with the results presented in Figure 11, which shows impedance increases consistent with significant mitochondrial depolarization for FCCP concentrations of $500 \mathrm{nM}$ or higher [40]. By contrast, low FCCP concentrations, of $50 \mathrm{nM}$ and $100 \mathrm{nM}$, cause only slight mitochondrial depolarization (Figure 12). FCCP at $200 \mathrm{nM}$ concentrations shows a greater increase in impedance, over a wider frequency range, indicating greater loss of mitochondrial membrane potential. Our results are thus consistent with FCCP being an effective mitochondrial uncoupler in a dose-dependent fashion $[44,45]$.

\section{Effects of dopamine on mitochondria}

Previous studies $[4,13,46]$ suggest that high dopamine concentrations induce mitochondrial dysfunction through a loss of membrane potential mediated by production of free radicals such as $\mathrm{NO}$ and $\mathrm{H}_{2} \mathrm{O}_{2}$. We therefore carried out a study to determine whether impedance methods would yield results consistent with those findings. Figure 13 shows that impedance raises quickly as higher dopamine concentrations are added to the mitochondrial suspension. This observation is consistent with a significant reduction of the mitochondrial membrane potential $\Delta \Psi_{m}$. Our results are consistent with findings [47] showing a 35\% decrease in state $3 \mathrm{O}$ uptake after $1 \mathrm{mM}$ dopamine incubation, if excess dopamine adversely affects the ability of respiratory complexes to build up a transmembrane proton motive force. Some results [47] show decreased mitochondrial RCR suggesting that high dopamine concentrations may also induce mitochondrial depolarization. Overall, the results in ref. [47] are

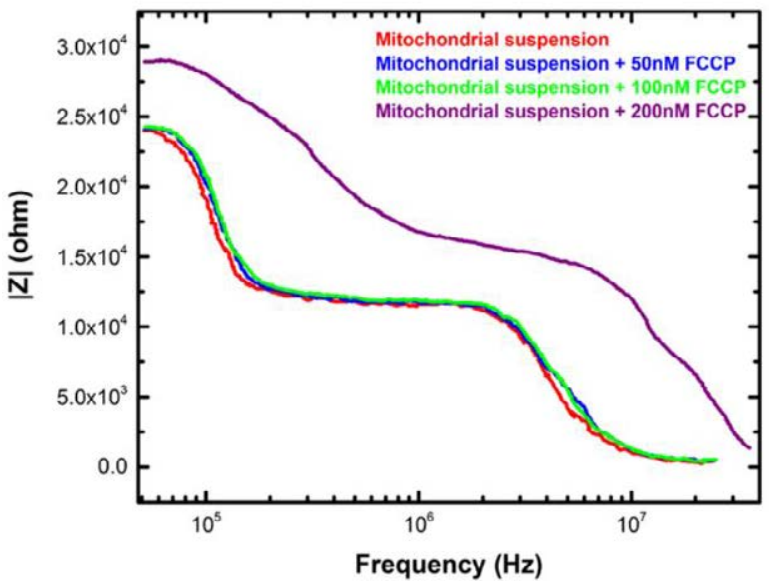

Figure 12: Changes in impedance caused by added FCCP concentrations during mitochondrial respiration. consistent with our findings that excess dopamine concentrations adversely affect mitochondrial membrane potential and function.

Figure 14 shows jumps in impedance at a fixed frequency $(100 \mathrm{kHz})$ when increasing concentrations of dopamine are added, indicating dose-dependent decreases in mitochondrial membrane potential. These results are thus consistent with the idea that high dopamine concentrations have deleterious effects on mitochondrial function, such as a decrease in mitochondrial respiration and/or depolarization of the mitochondrial inner membrane [47,48]. Similar results were reported in ref. [47,48], where investigation of $\mathrm{H}_{2} \mathrm{O}_{2}$ production by intact striatal mitochondria showed a significant increase after $0.5 \mathrm{mM}$ to 1 $\mathrm{mM}$ dopamine incubation. Increased production of $\mathrm{NO}$ was another interpretation as to why, dopamine might adversely affect respiratory chain complexes [47,48]. Evaluation of mitochondrial membrane potential showed that low physiological dopamine concentrations do not affect mitochondrial membrane potential, while high dopamine concentrations induce depolarization, as supported by Figure 14

Figure 15 compares impedance in which the mitochondrial suspension is incubated with $1 \mathrm{mM}$ of FCCP vs, $1 \mathrm{mM}$ of dopamine. The protonophore FCCP causes greater impedance increases, consistent with stronger mitochondrial depolarization as compared to the effects

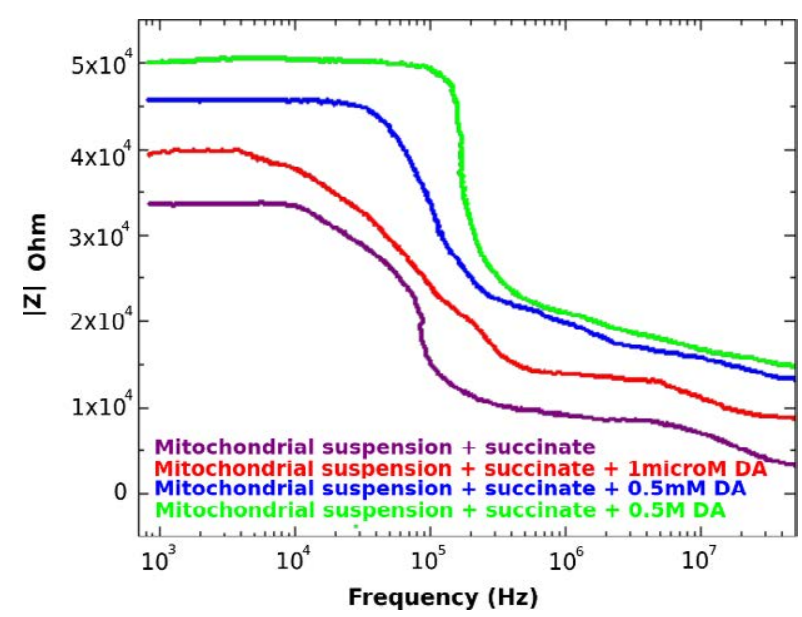

Figure 13: Impedance vs. frequency for 3 dopamine (DA) concentrations.

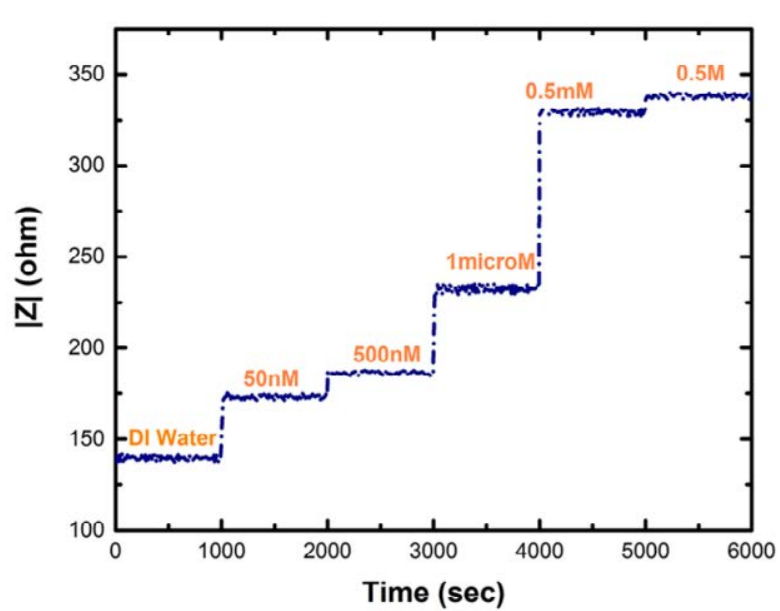

Figure 14: Impedance (at $100 \mathrm{kHz}$ ) vs. time for increasing concentrations of dopamine added to mitochondrial suspension. 
Citation: Mitra RC, Villagrán MYS, Maric S, Wosik J, Zagozdzon-Wosik W, et al. (2017) Evidence from Impedance Spectroscopy that Elevated Dopamine Reduces Mitochondrial Membrane Potential. J Biosens Bioelectron 8: 242. doi: 10.4172/2155-6210.1000242

Page 7 of 8

of dopamine [4]. This is further supported by Figure 16, which shows capacitance and conductance responses for different dopamine and FCCP concentrations.

\section{Conclusions}

Changes in neurotransmitter concentrations are known to affect the physiological and psychological properties of the brain [47-49]. Previous studies have shown that schizophrenia involves excessive amounts of dopamine in the frontal lobes, whereas too little dopamine in the motor areas is involved in Parkinson's disease [50-52]. The study reported here provides important clues about a possible additional role of dopamine in various neurodegenerative diseases, and could ultimately stimulate the development of improved treatments. In particular, our study provides further evidence that excess dopamine reduces mitochondrial membrane potential, either by inducing dysfunction in key respiratory complexes, uncoupling the mitochondrial inner membrane, or some combination of those effects. Ours results (Figures 13-16) show that dopamine has effects similar to, albeit less pronounced than, those of the ionophore FCCP.

High dopamine concentrations thus appear to have deleterious effects on mitochondrial function. This suggests a possible interpretation for contradictory roles of elevated dopamine in the manic and depressive states of bipolar disorder, where the depressive phase may be exacerbated by dopamine-induced mitochondrial dysfunction. Possible adverse effects of excess dopamine on cardiac mitochondria indicate the need for care in its use to treat, e.g., Parkinson's disease or heart failure. In summary, impedance spectroscopy has the potential to provide important insights on mitochondrial dysfunction and on the dynamic effects of various on mitochondrial function.

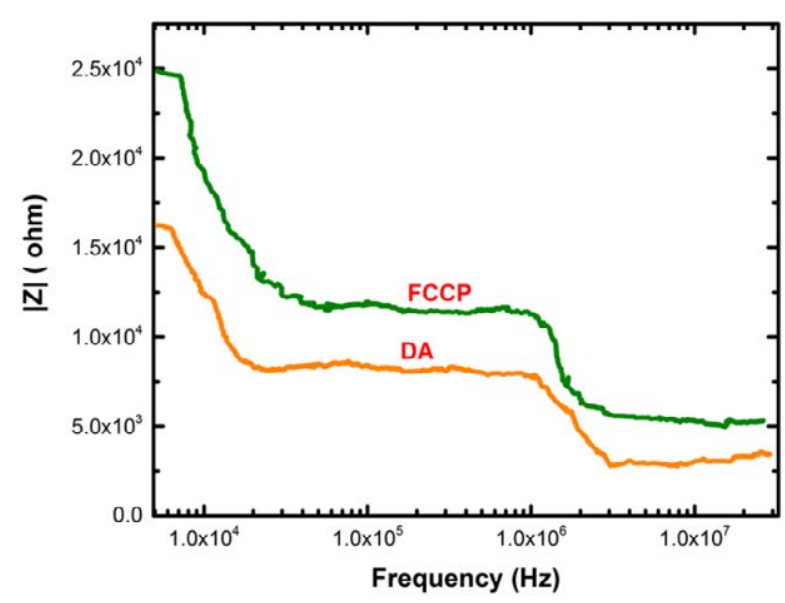

Figure 15: Comparison of impedance vs. frequency for dopamine and FCCP added to mitochondrial suspension.
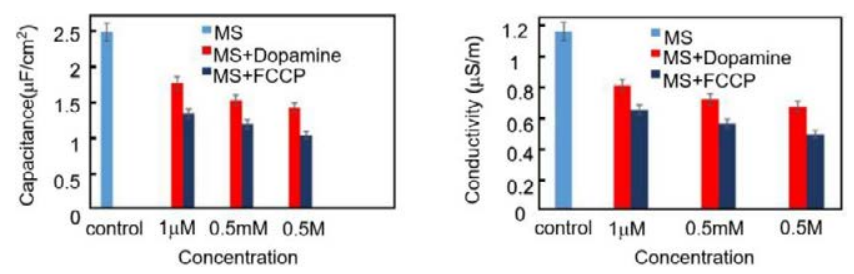

Figure 16: Comparison of capacitance and conductance of mitochondrial suspension (MS) after adding dopamine and FCCP.

\section{Acknowledgments}

This work was supported by the State of Texas through the Texas Center for Superconductivity at the University of Houston. The authors acknowledge the assistance of Nick Valenzuela and Lakshmi Reddy in the mitochondria extraction process, and helpful conversations with Pavithi Weerasinghe, Dale J. Hamilton, and Dong Cai.

\section{References}

1. Flippo KH, Strack S (2017) Mitochondrial dynamics in neuronal injury, development and plasticity. J Cell Sci 130: 671-681.

2. Murphy A (2000) Mitochondria in human disease. The Biochemist 22: 19-24

3. Li H, Sharma LK, Li Y, Hu P, Idowu A, Liu D, et al. (2013) Comparative bioenergetic study of neuronal and muscle mitochondria during aging. Free Radical Biology and Medicine 63: 30-40.

4. Czerniczyniec A, Bustamante J, Lores-Arnaiz S (2010) Dopamine modifies oxygen consumption and mitochondrial membrane potential in striatal mitochondria. Molecular and Cellular Biochemistry 341: 251-257.

5. Tiago V, Maia MJF (2017) An Integrative Perspective on the Role of Dopamine in Schizophrenia. Biological Psychiatry 18: 52-66.

6. Arnsten AF, Girgis RR, Gray DL, Mailman RB (2017) Novel dopamine therapeutics for cognitive deficits in schizophrenia. Biological Psychiatry 81 67-77.

7. Doenst T, Nguyen, TD, Abel ED (2013) Cardiac Metabolism in Heart Failure Implications beyond ATP production. Circulation Research 113: 709-724.

8. Leggio GM, Bucolo C, Platania CBM, Salomone S, Drago F (2016) Current drug treatments targeting dopamine D3 receptor. Pharmacology \& Therapeutics 165: $164-177$.

9. Zanettini R, Antonini A, Gatto G, Gentile R, Tesei S et al. (2007) Valvular Heart Disease and the Use of Dopamine Agonists for Parkinson's Disease. New England Journal of Medicine 356: 39-46.

10. De Marco T, Kwasman MMD, Debra LRN, Chatterjee K (1988) Dopexamine Hydrochloride in Chronic Congestive Heart Failure with Improved Cardiac Performance Without increased Metabolic Cost. American Journal of Cardiology 62: 57C-62C.

11. Elkayam U, Ng TMH, Hatamizadeh P, Janmohamed M, Mehra A (2008) Rena Vasodilatory Action of Dopamine in Patients With Heart Failure: Magnitude of Effect and Site of Action. Circulation 117: 200-205

12. Meiser J, Weindl D, Hiller K (2013) Complexity of dopamine metabolism. Cell Communication and Signaling 11: 34.

13. Czerniczyniec A, Bustamante J, Lores-Arnaiz S (2007) Dopamine enhances mtNOS activity: implications in mitochondrial function. Biochimica et Biophysica Acta (BBA)-Bioenergetics 1767: 1118-1125.

14. Chen L, Knowlton AA (2010) Mitochondria and heart failure: New insights into an ener-getic problem. Minerva Cardioangiologica 58: 213-229.

15. Rosca MG, Hoppel CL (2010) Mitochondria in heart failure. Cardiovascular Research 88: 40-50.

16. Labajova A, Vojtiskova A, Krivakova P, Kofranek J, Drahota Z et al. (2006) Evaluation of mitochondrial membrane potential using a computerized device with a tetraphenylphosphonium-selective electrode. Anal Biochem 353: 37-42.

17. Lin MT, Beal MF (2006) Mitochondrial dysfunction and oxidative stress in neurodegenerative diseases. Nature 443: 787-795

18. Padmaraj D, Pande R, Miller Jr JH, Wosik J, Zagozdzon-Wosik W (2014) Mitochondrial Membrane Studies Using Impedance Spectroscopy with Paralle pH Monitoring. PLoS ONE 9: 1-8.

19. Prodan C, Prodan E (1999) The dielectric behaviour of living cell suspensions Journal of Physics D: Applied Physics 32: 335.

20. Prodan C, Mayo F, Claycomb JR, Miller Jr JH, Benedik MJ (2004) Lowfrequency, low-field dielectric spectroscopy of living cell suspensions. Journal of Applied Physics 95: 3754-3756.

21. Prodan E, Prodan C, Miller Jr JH (2008) The Dielectric Response of Spherical Live Cells in Suspension: an Analytic Solution. Biophysical Journal 95: 4174 4182.

22. Chen J, Chengcheng Xue YZ, Bauer DC, Wu MH, Wang J (2015) Microfluidic 
Citation: Mitra RC, Villagrán MYS, Maric S, Wosik J, Zagozdzon-Wosik W, et al. (2017) Evidence from Impedance Spectroscopy that Elevated Dopamine Reduces Mitochondrial Membrane Potential. J Biosens Bioelectron 8: 242. doi: 10.4172/2155-6210.1000242

Impedance Flow Cytometry Enabling High-Throughput Single-Cell Electrical Property Characterization. Molecular Sciences 16: 9804-9830.

23. Sohn LL, Saleh OA, Facer GR, Beavis AJ, Allan RS, et al. (2000) Capacitance cytometry: Measuring biological cells one by one. Proceedings of the National Academy of Sciences of the USA 97: 10687-10690.

24. Vajrala V, Claycomb JR, Sanabria H, Miller JH Jr (2008) Effects of Oscillatory Electric Fields on Internal Membranes: An Analytical Model. Biophysical Journal 94: 2043-2052.

25. John CE, Jones SR (2007) In: Electrochemical Methods for Neuroscience (eds.), Michael AC, Borland LM, CRC Press/Taylor \& Francis, Boca Raton (FL).

26. Bruns D (2004) Detection of transmitter release with carbon fiber electrodes. Methods 33: 312-321.

27. Palmer JW, Tandler B, Hoppel CL (1977) Biochemical properties of subsarcolemmal and interfibrillar mitochondria isolated from rat cardiac muscle. Journal of Biological Chemistry 252: 8731-8739.

28. Itzhaki RF, Gill DM (1964) A micro-biuret method for estimating proteins Analytical Biochemistry 9: 401-410.

29. Frezza C, Cipolat S, Scorrano L (2007) Organelle isolation: functional mitochondria from mouse liver, muscle, and cultured filroblasts. Nature Protocols 2: 287-295

30. Gustafsson ÅB, Gottlieb RA (2008) Heart mitochondria: gates of life and death Cardio-vascular Research 77: 334-343.

31. Mourier A, Matic S, Ruzzenente B, Larsson NG, Milenkovic D (2014) The respiratory chain supercomplex organization is independent of COX7a2 isoforms. Cell Metabolism. 20: 1069-1075.

32. Bot C, Prodan C (2009) Probing the membrane potential of living cells by dielectric spectroscopy. European Biophysics Journal 38: 1049-1059.

33. Sanabria, H, Miller JH Jr (2006) Relaxation processes due to the electrodeelectrolyte interface in ionic solutions. Physical Review E 74: 051505.

34. Grimnes S, Rikshospitalet O, Schwan NHP (2002) Interface phenomena and dielectric properties of biological tissue. Encyclopedia of Surface and Colloid Science 20: 2643-2653.

35. Fricke H (1933) The Electric Impedance of Suspensions of Biological Cells Cold Spring Harbor Symposia on Quantitative Biology 1: 117-124.

36. Ellappan P, Sundararajan R (2005) A Simulation study of the electrical model of biological cells. J Electrostat 63: 297-307

37. Rustin P, Munnich A, Roetig A (2002) Succinate dehydrogenase and human diseases: new insights into a well-known enzyme. European Journal of Human Genetics 10:289-291.

38. Hollenbeck PJ, Bray D, Adams RJ (1985) Effects of the uncoupling agents FCCP and CCCP on the saltatory movements of cytoplasmic organelles. Cell Biology International Reports. 9: 193-199.
39. Park KS, Jo I, Pak K, Bae SW, Rhim H, et al. (2002) FCCP depolarizes plasma membrane potential by activating proton and $\mathrm{Na}+$ currents in bovine aortic endothelial cells. Pflugers Arch European Journal of Physiology 443: 344-352.

40. Brennan JP, Southworth R, Medina RA, Davidson SM, Duchen MR, et al. (2006) Mitochondrial uncoupling, with low concentration FCCP, induces ROS-dependent cardioprotection independent of KATP channel activation. Cardiovascular Research 72: 313-321.

41. Nicholls DG (2004) Mitochondrial membrane potential and aging. Aging Cell 3: $35-40$.

42. Brand MD, Nicholls DG (2011) Assessing mitochondrial dysfunction in cells. Biochemical Journal 435: 297-312.

43. Padmaraj D, Miller Jr JH, Wosik J, Zagozdzon-Wosik W (2011) Reduction of electrode polarization capacitance in low-frequency impedance spectroscopy by using mesh electrodes. Biosensors and Bioelectronics. 29: 13-17.

44. Feeney CJ, Pennefather PS, Gyulkhandanyan AV (2003) A cuvette-based fluorometric analysis of mitochondrial membrane potential measured in cultured astrocyte monolayers. Journal of Neuroscience Methods 125: 13-25.

45. Brennan JP, Berry RG, Baghai M, Duchen MR, Shattock MJ (2006) FCCP is cardioprotective at concentrations that cause mitochondrial oxidation without detectable depolarisation. Cardiovascular Research 72: 322-330.

46. Berman SB, Hastings TG (1999) Dopamine oxidation alters mitochondrial respiration and induces permeability transition in brain mitochondria. Journal of Neurochemistry 73 : 1127-1137.

47. Bains JS, Shaw CA (1997) Neurodegenerative disorders in humans: the role of glutathione in oxidative stress- mediated neuronal death. Brain Research Reviews 25: 335-358.

48. Gramigni E, Cozzi A, Pancani T, De Gaudio RA, Pellegrini-Giampietro DE, et al. (2006) Neuroprotective effects of propofol in models of cerebral ischemia: inhibition of mitochondrial swelling as a possible mechanism. Anesthesiology 104: 80-89.

49. Soane L, Kahraman S, Kristian T, Fiskum G (2007) Mechanisms of impaired mitochondrial energy metabolism in acute and chronic neurodegenerative disorders. Journal of Neuroscience Research 85: 3407-3415.

50. Bernheimer H, Birkmayer W, Hornykiewicz O, Jellinger K, Seitelberger $F$ (1973) Brain dopamine and the syndromes of Parkinson and Huntington Clinical, morphological and neurochemical correlations. Journal of the Neurological Sciences 20: 415-455.

51. Sternberg DE, VanKammen DP, Lerner P, Bunney WE (1982) Schizophrenia: dopamine beta-hydroxylase activity and treatment response. Science 216 1423-1425.

52. Ohara K, Ulpian C, Seeman P, Sunahara RK, Van Tol HH, et al. (1993) Schizophrenia: dopamine D1 receptor sequence is normal, but has DNA polymorphisms. Neuropsychopharmacology 8: 131-135. 